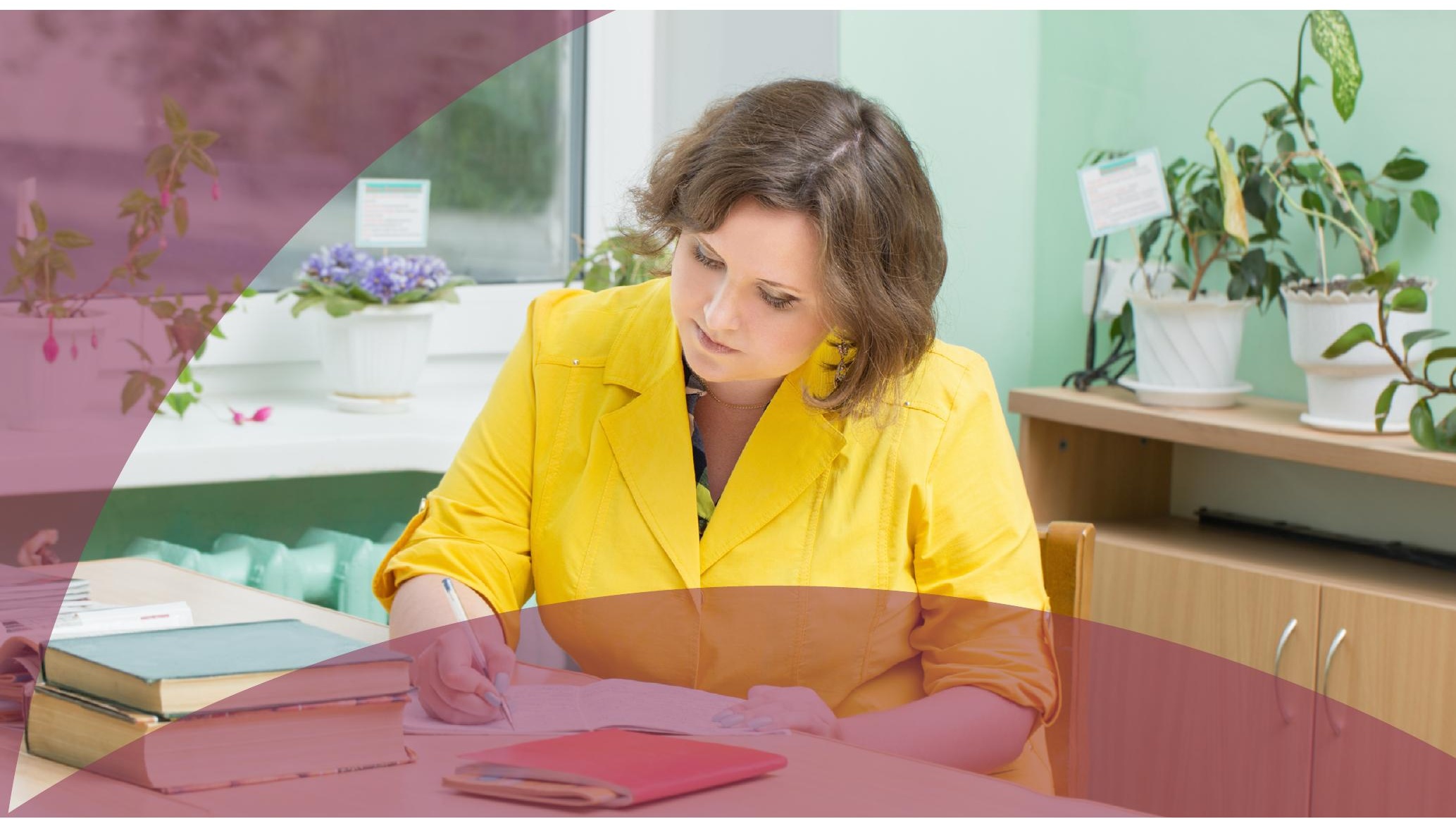

\title{
How effective are tools to help school staff better respond to young people who self-harm?
}

Self-harm among young people is a major public health concern, and now fears are mounting that the COVID-19 pandemic is having an added negative effect on young people's mental health. ${ }^{1,2}$ Although school staff are often the first to notice or hear about concerning self-harm behaviours, reports suggest that school staff often lack confidence and training in responding to this. ${ }^{3}$ Moreover, some studies have found that initial reactions to students' disclosure of self-harm are often negative. ${ }^{4}$ For this reason, Aureliane Pierret and colleagues at the University of Cambridge carried out a systematic review into the effectiveness, feasibility and

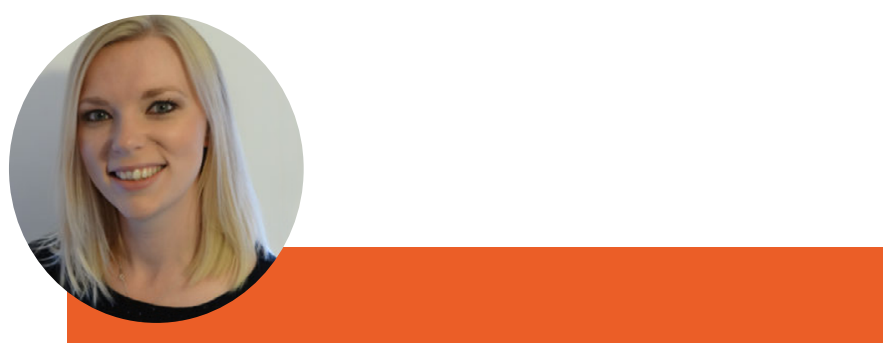

\section{Dr Jessica Edwards}

Jessica is a freelance editor and science writer, and has been writing for the Bridge since December 2017. 
acceptability of interventions and tools to support school staff to better respond to young people who disclose self-harm.

Pierret et al. identified eight studies for inclusion in their systematic review, with six reporting on educational and training interventions, and two on management and support tools, for schoolteachers to address self-harm in young people. These ranged from educational websites about self-harm in young people, to protocols outlining pathways to follow when responding to self-harm in schools. In some studies, training for school staff about this topic was given by experienced mental health professionals.

"All eight studies demonstrated effectiveness of these interventions and tools, with an increase in knowledge of school staff regarding how to respond to youth who are self-harming, and an increase in confidence when responding to these situations", explains Pierret. "In addition, there was generally a reduction in negative attitudes towards self-harm. Notably, however, these self-reported results do not necessarily reflect actual changes in practice, for which no data was collected". In addition, whilst data was lacking on the feasibility of these interventions, six studies did seem to have acceptable tools or interventions, with high rates of satisfaction and perceived benefit.

"Taking this forward, our findings suggest that a broad range of interventions can be effective and acceptable for schools to improve their staff's ability to respond to young people who are self-harming, and these can be tailored to the particular needs of each school and the population they serve", proposes Pierret. "Schools should be encouraged to adopt such trainings or educational tools to hopefully improve mental health outcomes for young people".

\section{Referring to:}

Pierret, A. et al. (2020), Review: Education and training interventions, and support tools for school staff to adequately respond to young people who disclose self-harm - a systematic literature review of effectiveness, feasibility and acceptability. Child Adolesc. Ment. Health. doi: 10.1111/camh.12436.

\section{References:}

${ }^{1}$ Holmes, E. A. et al. (2020), Multidisciplinary research priorities for the COVID-19 Pandemic: A call for action for mental health science. Lancet Psychiatry. 7, 547560. doi: 10.1016/S2215-0366(20)30168-1.

${ }^{2}$ Pierce, M. et al. (2020), A critical look at mental health surveys during COVID-19. Lancet. doi: 10.17863/CAM.54251.

${ }^{3}$ Berger E. et al. (2014), "We're Working in the Dark Here": Education needs of teachers and school staff regarding student self-injury. School Ment. Health. 6, 201-212. doi: 10.1007/s12310-013-9114-4.

${ }^{4}$ Berger, E. et al. (2014), Response and training needs of school staff towards student self-injury. Teach Teach. Educ. 44, 25-34. doi: 10.1016/j.tate.2014.07.013.

vif ACAMH CAMHS around the

Campfire: Expert discussion of this paper

: ACAMH Podcast: Creating mentally

$\square$ healthy schools, J Deighton

J'm JCPP Practitioner Review:

Effectiveness of indicated schoolbased interventions for adolescent depression and anxiety - a metaanalytic review, B Gee et al 